\title{
Eugenol Mediated Inhibition of Biofilm Formed by S. Aureus a Potent Organism for Pediatric Digestive System Diseases
}

\author{
KaiHua Ni \\ Soochow University \\ Danlei Cai \\ Soochow University \\ Jianhong Lu \\ Soochow University \\ Jianmei Tian ( $\nabla$ sudaert@sina.com ) \\ Soochow University
}

\section{Research Article}

Keywords: Eugenol, Ocimum tenuiflorum , Staphylococcus auresus, biofilm

Posted Date: June 28th, 2021

DOI: https://doi.org/10.21203/rs.3.rs-645217/v1

License: (c) (i) This work is licensed under a Creative Commons Attribution 4.0 International License.

Read Full License

Version of Record: A version of this preprint was published at Applied Biochemistry and Biotechnology on October 27th, 2021. See the published version at https://doi.org/10.1007/s12010-021-03682-x. 


\section{Abstract}

Ocimum tenuiflorum (KT) is a common ethno-botanical plant of the south-east Asia. The ethnic communities of these regions use the various parts of the plants specially the leaves for the treatment of various ailments like cold and flu, chronic infections and surface ailments. The leaves of these plants are consumed to act as immune boosters in the body. With this ethnical background we performed the antimicrobial and antibiofilm potential of the methanolic extract of Ocimum tenuiflorum (KT) against biofilm formed by $S$. aureus biofilm. The biofilm formed by $S$. aureus is a potent cause for the development of gastro-intestinal (GI) associated chronic infection. The extract from the KT leaf was analyzed using UV spectroscopy and HPLC to confirm the presence of the active ingredients present within the extract. The HPLC and GC-MS studies revealed the presence of eugenol and linalool in a greater proportion having the maximum drug like properties. It was observed that KT showed maximum inhibition of biofilm, protein and carbohydrate being present with the extracellular polymeric substance (EPS). Interestingly, the maximum inhibition to the quorum sensing (QS) and the genomic DNA, RNA content was reduced by eugenol and linalool in comparison to the plant extract. The studies were supported by in-silico interaction between eugenol and linalool with the QS proteins of $S$. aureus. The studies were further confirmed with microscopic studies SEM and FCM. The IR studies also confirmed much reduction in biofilm when treated with eugenol, linalool and KT with respect to the untreated sample.

\section{Introduction}

Microbial biofilm are complex association of sessile microorganisms attached to biotic or abiotic substrata with the help of extracellular polymeric substances (EPS) (1) forming a matrix and develops as a defense mechanism during extreme stressed conditions such as insufficient nutrient supply, extreme temperature, salt concentrations or $\mathrm{pH}$ variations etc. The reversible or irreversible binding to the solid surface with the help of EPS enables the microbes to spread its pathogenicity and adapts against antagonistic environment. EPS comprises of mostly water, extracellular DNA, RNA, proteins, lipids and is responsible for efficient supply of nutrients like glucose, amino acids and small molecules for the survival of biofilm associated microbes. The genetic diversity and inter-community interaction within the biofilm varies widely due to these stressed conditions forces the indwelling microbes to undergo a cell number density dependent sensing capabilities also known as quorum sensing (QS) that helps in the survival of the microorganisms within the biofilm niche by modulating the biofilm formation via up/down regulating cascade of genes. QS or cell-cell communication is performed by small bacterial signalling molecules known as autoinducers (Al) that detects the neighboring population density and triggers specific bacterial genes controlling factors such as sporulation, antibiotic production, competence, virulence, motility and biofilm formation (2). Though the signal transduction mechanisms, chemical nature of the signaling molecules and the target genes associated with various bacterial QS circuits differ widely in bacteria, ability of cell- cell communication and coordinated gene expression is a common factor in the bacterial society (3) 
In the context of medical world, biofilms of single and multiple species are widely found on surface of varied medical implants such as prosthetic heart valve, urinary catheters, dental implants etc (4). EPS matrix provides a protective barrier to the penetrating antibiotics and bacterial drugs which can act only on the planktonic (free floating cells) form of bacterial cells whereas the sessile (immobilize) cells develops resistance towards antibiotics and keep on propagating inside the biofilm (5) thus making the eradication of biofilm-forming bacteria more difficult and hence are of serious concern. Therefore, the conventional treatment strategies of medicines (6) required a novel approach with better pharmacological development. This involves discovery of new antimicrobial agents (7) such as traditional, ethnic and plant derived natural products having improved antimicrobial efficacy due to their secondary metabolites and possessing biofilm disrupting properties. Several researches have indicated towards the plant bioactive compounds having potent antibiofilm activity against varied bacterial infection (8-9) Bioactive compounds from plants are relatively safe to use as they do not harm the host tissues while acting the biofilm cells and also provide extra nutritional constituents enhancing the physiological and cellular activities in host organisms consuming them (10).

Staphylococcus aureus is a pathogenic and nosocomial agent that is responsible for the development of gastro-intestinal (GI) associated chronic infections and needs to be controlled properly (11). The present study is aimed at the use of ethanolic extract of Ocimum tenuiflorum (KT) leaves and its bioactive compounds as a potent antibacterial and antibiofilm agent against $S$. aureus mediated GI track infections.

\section{Materials And Methods}

\subsection{Microorganism and biofilm formation}

Overnight culture of Staphylococcus aureus ATCC 0352, bacterial strain was cultivated in $50 \mathrm{ml}$ of Luria Bertani broth $\left(\mathrm{pH} 7,37^{\circ} \mathrm{C}\right)$. Biofilm formation by $\mathrm{S}$. aureus was analyzed by using microplate assay method (12) by incubating the plates at temperature of $35^{\circ} \mathrm{C}$ for a period of 72 hour (13).

\subsection{Collection of Plant}

Ocimum tenuiflorum (KT) were collected from local areas and stored at room temperature.

\subsection{Extraction of Plant Extract}

The leaves of KT were pulverized in $95 \%$ ethanol with a mortar-pestle and kept for 24 hours following which the extract was filtered at kept at $4^{0} \mathrm{C}$ for further use (14).

\subsection{HPLC Analysis of Bioactive molecules}

Crude KT extract was fed into HPLC column for analytical HPLC analysis followed by identification of highest peaks in the chromatogram. The compound showing highest peak was isolated through preparative HPLC analysis and tested for antibacterial activity. 


\subsection{GC-MS Analysis}

The chromatographic analysis of the ethanolic extract of the KT leaves was done using GC Model-Trace GC Ultra, MS model- POLARISQ (Thermoscientific). The data obtained were searched using NIST Library and compared by using Wiley Spectral library search programme (15).

\subsection{Determination of Minimum Inhibitory Concentration (MIC) and Minimum Bactericidal Concentration (MBC)}

Ethanolic extract of KT leaves were centrifuged at 10,000 rpm for 10 minutes and the supernatant was used as antibiofilm agent. The concentration of crude solution of Tetracyclin (500mg dissolved in $10 \mathrm{~mL}$ of deionized water) was adjusted by serial dilution method for control experiments. The MIC was determined (16) by disc diffusion technique and MBC was determined by broth dilution method by dissolving 2,3,5-triphenyl tetrazolium salt (TTC)

\subsection{Minimum Biofilm Eradication Concentration Analysis (MBEC)}

Liquid cultures $(100 \mu \mathrm{L}$ of LB broth) of $S$. aureus were grown in 96 well polystyrene flatbottom tissue culture microplates for a period of 72 hours followed by discarding the broth and washing the cells with $0.1 \%$ normal saline $(\mathrm{w} / \mathrm{v})$. This was followed by the addition of the ethanolic extract of KT and tetracyclin keeping one well blank as control. Concentration of viable bacterial cells was measured based on the ability of the living cells to convert yellow tetrazolium salt to purple formazon product. The percentage inhibition was calculated using the equation (17):

\section{$\left[1-\frac{A 570 \text { of the test }}{\text { A570 of non }- \text { treated control }} \times 100\right]$}

\subsection{Spectroscopic measurements of $\mathrm{N}$-acyl-homoserine lactone concentration (AHL) and Quorum sensing assay}

$5 \mathrm{~mL}$ of Luria Bertani Broth (Himedia) were inoculated with Staphylococcus aureus ATCC 0352 overnight at $37^{\circ} \mathrm{C}$ for 24 hours. From this cultured broth, $2 \mathrm{~mL}$ of bacterial culture was transferred for centrifugation at 10,000 rpm for 15 minutes. The supernatant was collected and filtered using membrane filter of diameter $0.2 \mu \mathrm{m}$ to remove any traces of cell debris. The filtrate was treated with ethyl acetate with mild shaking condition and was subsequently allowed to stand for 5 minutes. This resulted into separation of upper and lower immiscible layer. This method of liquid-liquid extraction (LLE) helps the upper layer to be separated off and dried at oven at $40^{\circ} \mathrm{C} .40 \mu \mathrm{L}$ of LLE sample (18) was inoculated into wells of 96-well polystyrene flatbottom tissue culture microplates which was previously supplemented with $50 \mu \mathrm{L}$ of a 1:1 mixture of hydroxyl amine (2M): $\mathrm{NaOH}$ (3.5M). This was followed by addition of equal amount of 1:1 mixture of ferric chloride ( $10 \%$ in $4 \mathrm{M} \mathrm{HCl}): 95 \%$ ethanol. A dark brown color was shown from all of the samples containing lactone compounds. Optical density (OD) was measured at $520 \mathrm{~nm}$. Precaution must be taken in maintaining a steady acidic $\mathrm{pH}$ for monitoring $\mathrm{AHL}$. This is due to the inactivation of $\mathrm{AHL}$ 
under alkaline conditions by $\mathrm{pH}$-dependent lactonolysis. During lactonolysis, homoserine lactone ring is hydrolyzed to open ring form corresponding to N-acylhomoserine.

\subsection{Isolation and purification of Extracellular polymeric substance (EPS)}

EPS matrix comprises of proteins, polysaccharides, nucleic acids, multivalent cations, lipids, and inorganic particles (19) and forms about $50-90 \%$ of the total biofilm mass (20).

\subsubsection{Carbohydrate estimation}

Treatment with plant extracts results into disruption of EPS matrix elements. The bactericidal effects of $\mathrm{KT}$ extracts were tested on the amount of carbohydrate present in bacteria after treating with KT extracts and control. $100 \mu \mathrm{l}$ of EPS sample pretreated with KT extract was diluted with $900 \mu \mathrm{l}$ of distilled water in test tube. This was followed by $5 \mathrm{~mL}$ of $98 \%$ concentrated $\mathrm{H}_{2} \mathrm{SO}_{4}$ and $1 \mathrm{ml}$ of phenol. The reaction mixture was kept undisturbed till the colorless solution turned yellow in color. The blank was set with $\mathrm{H}_{2} \mathrm{SO}_{4}$ and Phenol. The OD was measured at $490 \mathrm{~nm}(21)$.

\subsubsection{Protein estimation}

$100 \mu$ l of EPS sample pretreated with KT extract was diluted with $900 \mu$ l of distilled water in test tube. This was followed by adding $5 \mathrm{ml}$ of $0.1 \mathrm{mM}$ Copper sulphate resulting into a blue solution. The test tubes were incubated for 10 minutes in water bath at $60^{\circ} \mathrm{C}$ followed by addition of $500 \mu$ l Folin reagent and incubated for $1 \mathrm{hr}$ in $37^{\circ} \mathrm{C}$. This results into transformation of the turbid sky blue color into transparent dark blue coloured solution. The OD was measured at 700nm. The blank was set with copper sulphate Folin solution without the sample.

\subsubsection{Nucleic acid estimation}

$200 \mathrm{ul}$ of EPS sample pretreated with KT extract were put in the test tubes followed by addition of 600uL of $99.9 \% \mathrm{w} / \mathrm{v}$ ethanol and incubated for $2 \mathrm{hr}$ in $37^{\circ} \mathrm{C}$. The OD was measured at $260 \mathrm{~nm}$. The blank was set with ethanol (22).

\subsection{Determination of the viability count of the biofilm forming cells}

Viablility of bacterial cells is best measured by monitoring the bacterial growth kinetics in presence and absence of antimicrobials. For monitoring the viability of biofilm forming $S$. aureus cells, liquid cultures of S. aureus in LB broth having $0.1 \%$ chitin flakes $(\mathrm{w} / \mathrm{v})$ were incubated for a period of 72 hours. This was followed by discarding the media and washing it with $0.1 \%(\mathrm{w} / \mathrm{v})$ normal saline so as to eliminate the planktonic groups of cells. The sessile cells within the biofilm were treated with KT extracts and the growth was determined by UV-Visible spectrophotometer at $590 \mathrm{~nm}$ at intervals of time.

\subsection{Determination of Biofilm formation by Scanning Electron microscopy (SEM)}


S. aureus biofilms were grown on chitin flakes submerged in LB broth for a period of 72 hours in test tubes. This was followed by washing with $0.9 \% \mathrm{NaCl}$ to remove planktonic cells and subsequently challenged with the KT extract for 2 hours. A single chitin was recovered from the test tube and kept in $2.5 \%$ glutaraldehyde for 20 min followed by dehydration with ethanol overnight. The dried biofilms were coated with gold and visualized under a scanning electron microscope (Model-ZEISS EVO-MA 10) (23).

\subsection{Protein structure preparation and molecular docking}

X-ray crystallographic structures of $S$. aureus quorum sensing protein (PDBID- 3TIP) having good resolution of about 1.5-2A was obtained the Protein Data Bank (PDB). Any co-crystallized ligand attached to the above protein was removed from the binding site and this modified protein structure was further used for molecular docking interaction studies with Autodock 4.2 software. Bioactive compounds from Ocimum tenuiflorum were found from PubChem Database after it has been detected in GC-MS chromatogram profiles. Ligands were obtained in SDF format and then converted into PDB format using OpenBabel for its easy recognition in Autodock software. Autodock Tools, Gasteiger charges and solvation terms were assigned to the modified protein to compute its bulk and surface properties. Genetic algorithm (GA) method was used to compute the cluster of probable conformations of the ligand at the protein binding site. Standard docking settings were applied and the energetically most favorable binding poses (lowest docked energy) are taken to obtain the best conformation (24-25).

\subsection{CABS-flex analysis}

It is an efficient modeling procedure for fast simulations of protein structure flexibility. It is based on a well-established coarse-grained protein modeling tool that generates protein dynamics at highly reduced ( 3 orders of magnitude) computational cost, although with some decrease in resolution. A consensus view of protein near-native dynamics is obtained from 10-nanosecond MD simulations (all-atom, explicit water, for all protein metafolds using the four most popular force-fields). The docked output file is loaded in the CABS-flex 2.0 online server (http://biocomp.chem.uw.edu.pl/CABSflex2) that helps predict the interaction of bioactive compounds of KT with biofilm forming proteins of $S$. aureus. (26)

\subsection{LIGPLOT analysis}

The docked protein-ligand complex is used for identification of the nearest amino acid residues that closely interacts with the ligand. 2-D representations of protein-ligand complexes from standard PDB file input are uploaded in LIGPLOT and it generates images closely associated residues of the bacterial QS protein (27).

\subsection{Statistical analysis}

All the experimental outcomes obtained were performed in triplicate analysis and depicted as mean \pm SE (standard deviation).

\subsection{Chemicals}


All chemicals used were obtained from Merck, Germany and HIMEDIA, India. The chemicals were of analytical grade and used without any further purification.

\section{Results And Discussion}

\subsection{GC-MS Analysis of the Ethanolic Extract of Ocimum tenuiflorum (KT)}

Gas chromatographic analysis of ethanolic extract of KT was performed and the mass chromatogram of the unknown bioactive compounds were identified by comparing with the chromatogram of known compounds already stored in NIST (National Institute Standard and Technology) library. The compound name, molecular weight, molecular formula and peak area of the test sample were determined. Various bioactive compounds of KT identified from GC-MS chromatogram are listed in Table 1. The major components found were gallic acid, $p$-coumaric acid, cinnamic acid, catechol, caffeic acid, 3,4dimethoxycinnamic acid, luteolin, diosmetin, kaempferol, apigenin, rosmarinic acid, genistein, eucalyptol, camphor and eugenol (28) Presence of various compounds eluted at different retention times were detected once the large compound fragments into small compounds resulting into characteristic peaks at different $\mathrm{m} / \mathrm{z}$ ratios which can be further compared with the standard NIST library. Among these detected bioactive compounds, few possesses the potential to act as future drug candidate molecules which can be further tested for their biological activities in vitro and in vivo.

\subsection{Quorum Sensing Assay}

Quorum sensing (QS) is a cell population density dependent signaling mechanism developed by microorganisms during biofilm formation and maturation. Bacterial cells striving within the EPS matrix of biofilm can efficiently sense the neighbouring cells by a series of small signaling molecules such as aceyl-homoserine lactones (AHL), small peptides, autoinducers (AI) regulated by a complex genetic machinery. Formation of biofilm is directly proportional to the AHL secretion by the microorganisms. Thus quantification of AHL serves an essential purpose for the detection of QS mechanism. Figure 3 shows that the QS are drastically reduced in comparison to control (S. aureus) at OD at $520 \mathrm{~nm}$ which is $0.823 \pm 0.073$ to $0.382 \pm 0.031$ when treated with KT extract and $0.513 \pm 0.026$ when treated with tetracycline. We detected a significant decrease in QS activity in presence of ethanolic extracts of KT as compared to tetracyclin treated and control sample indicating the anti-quorum sensing and antibiofilm efficacy of KT (29).

\subsection{Effect of ethanolic extract of Ocimum tenuiflorum upon various components of EPS}

Biofilm comprises of sessile microbial colonies embedded in a slimy layer of exopolymeric substances known as EPS which consists majorly of water, carbohydrate, proteins, lipids and nucleic acids. The presence and absence of the challenge of ethanolic extract of KT were estimated upon various EPS components. It was observed that components of EPS viz. protein, carbohydrates and nucleic acids were largely reduced by the challenge of ethanolic extract of KT. The carbohydrate content within EPS of $S$. aureus biofilm were effected maximum by the ethanolic extract of $K T$ for a time challenge of 2 hours 
showing $56.2 \%$ with respect to control $(0.168 \pm 0.023$ to $0.072 \pm 0.011 \mathrm{mM})$. The protein and nucleic acid content of $S$. aureus EPS matrix were reduced by the ethanolic extract of $K T$ for a time challenge of 2 hours showing $21.8 \%$ and $44.3 \%$ reduction with respect to control $(0.138 \pm 0.013$ to $0.108 \pm 0.011 \mathrm{mM})$ and $(0.183 \pm 0.016$ to $0.102 \pm 0.041 \mathrm{mM})$ respectively (Figure 4$)$. The reduction in protein, carbohydrate or nucleic acid content indicates towards the disintegration of EPS matrix in comparison to the stable and intact EPS matrix of the control sets (30). All the data were Mean \pm SE. The data were statistically significant.

\subsection{Minimum Biofilm Eradication Concentration (MBEC) determination assay}

MBEC assay was performed to calculate the potency of KT in successful eradication of S. aureus biofilm. It provides information regarding the minimum dosage required for clearance of the sessile bacterial colony formed on various biotic/abiotic surfaces. From Figure 5 it can be concluded that S.aureus were eradicated by $73.81 \pm 8.97 \%$ in the challenge of Ocimum tenuiflorum $(K T)$ at an inhibitory concentration of and $51.45 \pm 5.13 \%$ in the presence of antibiotic Tetracycline

\subsection{Viability Count of the biofilm cells in the presence or absence of the KT}

The viability count of the S.aureus cells were found to decrease growth (log $\mathrm{CFU} / \mathrm{ml}$ ) for a time challenge of 0 to 48 hours with a maximum effect by Ocimum tenuiflorum (KT) from $6.18 \pm 0.014$ to $6.02 \pm 0.02$ in comparison to control $(6.21 \pm 0.03$ to $6.61 \pm 0.02)$ and tetracyclyine $(6.19 \pm 0.005$ to $6.056 \pm 0.018)$. It was also observed that upon removal of challenge of KT extract lead to a negligible revival from log $\mathrm{CFU} / \mathrm{ml}$ $6.22 \pm 0.015$ to $6.029 \pm 0.024$ in comparison to the control that showed a continuous growth from log $\mathrm{CFU} / \mathrm{ml} 6.18 \pm 0.03$ to $6.58 \pm 0.013$ and $\log \mathrm{CFU} / \mathrm{ml} 6.198 \pm 0.005$ to $6.086 \pm 0.018$ on removing the challenge of standard antibiotic (Figure $6 \mathrm{a}$ ) and $\mathrm{b}$ )). The revival studies were performed after allowing the cultures to grow for 24 hours after removing the challenge of 0 to 48 hours. The data were statistically significant.

\subsection{Microscopic studies of biofilm}

The effect of time challenge of KT extract upon the S. aureus biofilm topology was observed by scanning electron microscopy. The control sample (Figure 18b) comprises of well-developed and mature biofilm cells adhered on the chitin surface whereas a dispersed and partially distorted biofilm assembly was observed on the KT extract treated samples (Figure 18c). This was further supported by fewer bacterial colonies in the biofilm treated with KT extract. SEM micrograph image and QS assay suggests that ethanolic extract of Ocimum tenuiflorum directly effects the biofilm development and maturation by influencing the flagellum driven motility which is necessary for attachment to the substratum via swimming and swarming motion (31).

\subsection{Docking between biofilm forming protein (3TIP) of S.aureus with potent bioactive compounds from Ocimum tenuiflorum (KT)}

The bioactive molecules identified from GC-MS spectrograph of ethanolic extract of KT were used for understanding the mechanism of binding with the biofilm forming protein of $S$. aureus (3TIP) with the 
help of molecular docking. In-silico docking analysis helps predict the binding sites and major interacting amino acid residues with the phytocompounds alongwith the binding interaction energies (32). This analysis helps predict the best docked poses based on minimum binding energies alongwith the tentative interaction viz. $\mathrm{H}$-bonds, electrostatic interactions or pi-pi interactions taking place between the amino acid residues and the phytocompounds enabling the prediction of few putative lead molecules that can be looked for future drug compounds. Binding energy is released when drug molecule associated with a target, which causes lowering of the overall energy of the complex. This release of binding energy compensates for any transformation of the ligand from its energy minimum to its bound conformation with the protein. Therefore, the greater the energy released (i. e., the greater the negative value), on binding of a ligand to the protein, greater is the propensity of the ligand to associate with that protein. From the docking results obtained, it is observed that rosmarinic acid, ursolic acid, oleanolic acid show the highest binding energy $\left(\Delta \mathrm{G}^{\circ}\right)$ with the Staphylococcus aureus as compared to other bioactive compounds present (Table 2). The docked results were further analysed with Ligplot software for identifying the key residues in the protein and CABs-flex server for performing the 20ns MD simulation.

From the Ligplot analysis, various amino acids that are interacting with the biofilm forming bacterial proteins (3TIP) are observed (33). These amino acids interact with the proteins via different types of bond like hydrophobic bonds, hydrogen bonds, pi-pi stacking bonds and salt bridges. Also, from the online server called PLIP (Protein-Ligand Interaction Profiler) the distances of the bioactive compounds from their binding pockets is obtained. These distances determine the type of bond each amino acid residue has with the bioactive compounds (ligands). Upon comparison of the results obtained from both the Ligplot software and PLIP server, it can be concluded that results obtained from them are almost identical and that, both the software and server have given accurate results. From the results obtained, it can be seen that rosmarinic acid and linalool have highest number of bonds meaning that they have highest amount of stability while interacting with the biofilm forming proteins of Staphylococcus aureus (Table 3)

From the CABS FLEX analysis (Table 4), it is observed that almost all the bioactive compounds show significant variations in their interaction with biofilm forming proteins of Staphylococcus aureus (3TIP). From these variations, the interaction of the biofilm forming proteins with and without their ligands (bioactive compounds) is observed. Most significant variations are observed in Staphylococcus aureus (3TIP).

\section{Conclusion}

Biofilms are formed by sessile microorganisms adhered to biotic/abiotic surfaces and are well protected within a self-secreted exopolymeric matrix consisting of proteins, carbohydrates, lipids, nucleic acids, small signaling molecules etc. Biofilm formation is a defense strategy developed by the microbial cells under stress situation such as adverse environments, host immune responses or presence of antimicrobials that lead to enhanced immune responses of the microbial cells persisting within the biofilm matrix. This matrix is also responsible for improved resistance towards the antimicrobial agents leading to the development of the phenomenon known as antimicrobial resistance (AMR). This has led to 
a surge in the development of resistance towards a wide range of antibiotics. Thus, alternative treatment strategies are an urgent requirement for fighting the ever increasing problems of AMR. One plausible mechanism involves biofilm reduction with phytochemicals of herbal plants of ethno-botanic origin. In the present work, we have investigated the antibiofilm and antimicrobial effect of ethanolic extract of the whole plant of Ocimum tenuiflorum (KT) which is known to contain a vivid range of bioactive compounds such as essential oils, flavonoids, terpenes etc against Staphylococcus aureus ATCC 0352 biofilm responsible for development of gastro-intestinal (GI) associated chronic infections in comparison to common antibiotic tetracycline. KT consists of bioactive compounds such as eugenol, apigenin, urosolic acid, carvacrol, linalool caryophyllene, carvicol etc. which brings subsequent reduction in the sessile cell population density as depicted by the slow rate of bacterial revival after withdrawal of antimicrobial stress of plant extracts. KT extracts is also successful in diminishing the bacterial QS mechanism by effecting the secretion of signaling molecules like AHLs. Molecular docking of $\mathrm{S}$. aureus biofilm protein with bioactive compounds showed good binding propensity. However, future studies relating to biofilm eradication ability, mechanism of action and in vivo potencies in controlling bacterial propagation are required to elucidate the potential usefulness of these herbal compounds as drug molecules.

\section{Declarations}

\section{Ethical Approval}

Not Applicable

\section{Consent to Participate}

All authors has their consent to participate

\section{Consent to Publish}

All authors has their consent to publish their work

\section{Authors Contributions}

All authors contributed equally

\section{Funding}

No funding of any kind has been received for the present work. Data have been generated as part of the routine work.

\section{Competing Interests}

The authors don't have any conflict of interest

\section{Availability of data and materials}




\section{References}

1. Donlan RM, Costerton JW. Biofilms: Survival mechanisms of clinically relevant microorganisms. ClinMicrobiol Rev 2002;15(2):167-93

2. Caicedo JC, Villamizar S, Ferro JA (2017) Quorum sensing, its role in virulence and symptomatology in bacterial citrus canker. https://doi.org/10.5772/66721

3. Nag M., Lahiri D., Ghosh A., Das D., Ray R.R. (2021) Quorum Sensing. In: Ray R.R., Nag M., Lahiri D. (eds) Biofilm-Mediated Diseases: Causes and Controls. Springer, Singapore. https://doi.org/10.1007/978-981-16-0745-5_2

4. Khatoon Z, McTiernan CD, Suuronen EJ, Mah TF, Alarcon El. Bacterial biofilm formation on implantable devices and approaches to its treatment and prevention. Heliyon. 2018;4(12):e01067. Published 2018 Dec 28. doi:10.1016/j.heliyon.2018.e01067

5. Costerton, J. W., Stewart, P. S., \& Greenberg, E. P. (1999). Bacterial biofilms: a common cause of persistent infections. Science, 284(5418), 1318-1322

6. Chadha T (2014) Bacterial Biofilms: Survival Mechanisms and Antibiotic Resistance. J BacteriolParasitol 5:190

7. Firdaus Jahan, Rubina Lawrence*, Vinod Kumar and Mohd.Junaid ,J. Chem. Pharm. Res., 2011, 3(4): 777-789

8. Mickymaray S. Efficacy and Mechanism of Traditional Medicinal Plants and Bioactive Compounds against Clinically Important Pathogens. Antibiotics (Basel). 2019;8(4):257. Published 2019 Dec 9. doi:10.3390/antibiotics8040257

9. Famuyide, I.M., Aro, A.O., Fasina, F.O. et al. Antibacterial and antibiofilm activity of acetone leaf extracts of nine under-investigated south African Eugenia and Syzygium (Myrtaceae) species and their selectivity indices. BMC Complement Altern Med 19, 141 (2019). https://doi.org/10.1186/s12906-019-2547-z).

10. Lahiri, D., Dash, S., Dutta, R. et al.Elucidating the effect of anti-biofilm activity of bioactive compounds extracted from plants. J Biosci44, 52 (2019). https://doi.org/10.1007/s12038-019-98684

11. Flaxman, A., van Diemen, P.M., Yamaguchi, Y. et al. Development of persistent gastrointestinal S. aureus carriage in mice. Sci Rep 7, 12415 (2017). https://doi.org/10.1038/s41598-017-12576-0

12. Lahiri, D ; Nag M and Ray R.R (2018) Comparative Study of the Antibiofilm Effect of plant extracts and antibiotic on Staphylococcus aureus ATCC 0352:International journal of basic and applied research. 8 (10):419-427

13. Aduse-Opoku, J., Slaney, J. M., Hashim, A., Gallagher, A., Gallagher, R. P., Boutaga, K., Laine, M. L., Curtis, M. A., Rangarajan, M. and Van Winkelhoff, A. J. (2006). Identification and characterization of 
the capsular polysaccharide (K-antigen) locus of Porphyromonasgingivalis.Infection and Immunity $74,449-60$

14. Cassandra L. Quave, Miriam Este'vez-Carmona, Cesar M. Compadre, Gerren Hobby, Howard Hendrickson, Karen E. Beenken, Mark S. Smeltzer, 2012, "Ellagic Acid Derivatives from Rubus uimifolius inhibit Staphylococcus aureus Biofilm Formation and Improve Response to Antibiotics". PLoS ONE 7, e28737

15. Abirami P, Rajendran A. GC-MS determination of bioactive compounds of Indigo feraaspalathoides. J Nat Prod Plant Res. 2011;1(4):126-30

16. AL-Saadi', Zainab Nashaat (2016) Estimation of Minimum Inhibitory Concentration (MIC) and Minimum Bactericidal Concentration (MBC) of Cell-Free Extracts of BifidobacteriumSpecies Against Methicillin-Resistant Staphylococcus aureusin vitro.American Journal of Biomedical and Life Sciences, Volume 4, Issue 5, October 2016, Pages: 75-80

17. Tang HJ, Chen CC, Ko WC, Yu WL, Chiang SR, Chuang YC(2011).Int J Antimicrob Agents, In vitro efficacy of antimicrobial agents against high-inoculum or biofilm embedded methicillinresistant Staphylococcus aureus with vancomycin minimal inhibitory concentrations equal to $2 \mu \mathrm{g} / \mathrm{ml}$ (VA2MRSA).. 2011; 38(1):46-51

18. Yang YH, Lee TH, Kim J, Kim EJ, Joo HS, Lee CS, Kim BG. High-throughput detection method of quorum-sensing molecules by colorimetry and its applications. Analytical Biochemistry 2006; 356(2): 297-299

19. Mayer C, Moritz R, Kirschner C, Borchard W, Maibaum R, Wingender J, Flemming HC (1999) The role of intermolecular interactions: studies on model systems for bacterial biofilms. Int J Biol Macromol 26:3-16

20. Donlan, R. M. (2002). Biofilms: Microbial Life on Surfaces. Emerging Infectious Diseases, 8(9), 881890. doi:10.3201/eid0809.020063

21. Andersson, S., Dalhammar, G., Land, C., and Rajarao, G. (2009). Characterization of extracellular polymeric substances from denitrifying organism Comamonas denitrificans. Appl. Microbiol. Biotechnol. 82, 535-543

22. Lahiri, D., Nag, M., Dutta, B. et al.Catechin as the Most Efficient Bioactive Compound from Azadirachta indica with Antibiofilm and Anti-quorum Sensing Activities Against Dental Biofilm: an In Vitro and In Silico Study. App/ Biochem Biotechno/193, 1617-1630 (2021). https://doi.org/10.1007/s12010-021-03511-1

23. Singh, V. K., Kavita, K., Prabhakaran, R., and Jha, B. (2013). Cis-9-octadecenoic acid from the rhizospheric bacterium Stenotrophomonas maltophilia BJ01 shows quorum quenching and antibiofilm activities. Biofouling 29, 855-867

24. Trott O., Olson A.J.,AutoDockVina: improving the speed and accuracy of docking with anew scoring function, efficient optimization, and multithreading.JComput Chem. 31:455-61(2010

25. Morris G.M., Goodsell, D. S., Halliday, R. S.,Huey,R., Hart, W. E., Belew, R. K.,Olson, A. J.,Automated docking using a Lamarckian genetic algorithm and an empirical binding free energy function. $J$ 
Comput Chem. 19: 1639-1662(1998

26. Kurcinski, M., Oleniecki, T., Ciemny, M.P., Kuriata, A., Kolinski, A. and Kmiecik, S. (2018). CABS-flex standalone: a simulation environment for fast modeling of protein flexibility. Bioinformatics, bty 685

27. Laskowski R A, Swindells M B (2011). LigPlott: multiple ligand-protein interaction diagrams for drug discovery. J. Chem. Inf. Model., 51, 2778-2786

28. Kaur, P., Dhull, S.B., Sandhu, K.S. et al. Tulsi (Ocimum tenuiflorum) seeds: in vitro DNA damage protection, bioactive compounds and antioxidant potential. Food Measure 12, 1530-1538 (2018). https://doi.org/10.1007/s11694-018-9768-6

29. Lahiri D., Nag M., Dutta B., Dash S., Ghosh S., Ray R. (2021) Synergistic Effect of Quercetin with Allicin from the Ethanolic Extract of Allium cepa as a Potent AntiQuorum Sensing and Anti-Biofilm Agent Against Oral Biofilm. In: Ramkrishna D., Sengupta S., Dey Bandyopadhyay S., Ghosh A. (eds) Advances in Bioprocess Engineering and Technology. Lecture Notes in Bioengineering. Springer, Singapore. https://doi.org/10.1007/978-981-15-7409-2_7

30. Lahiri, D., Nag, M., Dutta, B., Dey, S., Mukherjee, D., Joshi, S.J. and Ray, R.R. (2021), Antibiofilm and Anti-Quorum sensing Activities of Eugenol and Linalool From Ocimum tenuiflorum against Pseudomonas aeruginosa Biofilm. Journal of Applied Microbiology. Accepted Author Manuscript. https://doi.org/10.1111/jam.15171

31. Nicolás Gómez-Sequeda, Marlon Cáceres, Elena E. Stashenko, William Hidalgo, Claudia Ortiz, Antimicrobial and Antibiofilm Activities of Essential Oils against Escherichia coli 0157:H7 and Methicillin-Resistant Staphylococcus aureus (MRSA), Antibiotics 2020, 9, 730; doi:10.3390/antibiotics9110730

32. Cordeiro L, Figueiredo P, Souza H, et al. Antibacterial and Antibiofilm Activity of Myrtenol against Staphylococcus aureus. Pharmaceuticals (Basel). 2020;13(6):133. Published 2020 Jun 25. doi:10.3390/ph13060133

33. Chemmugil P, Lakshmi P, Annamalai A. A Multidisciplinary Study to Evaluate the Anti-quorum Sensing Ability of Phyto-compounds in Ruellia patula Jacq. Avicenna J Med Biotechnol. 2019;11(1):48-58

\section{Tables}

Due to technical limitations, table 1 is only available as a download in the Supplemental Files section.

\section{Figures}




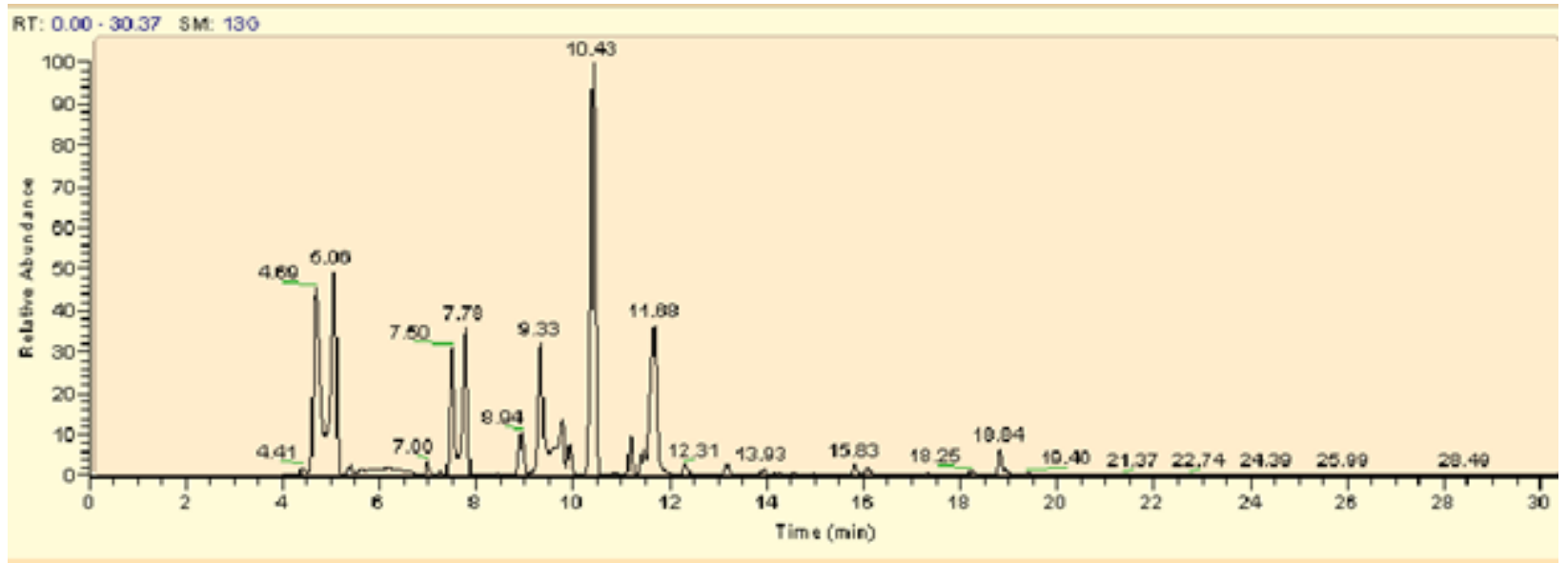

Figure 1

GC-MS chromatogram of ethanolic extract of Ocimum tenuiflorum (KT)

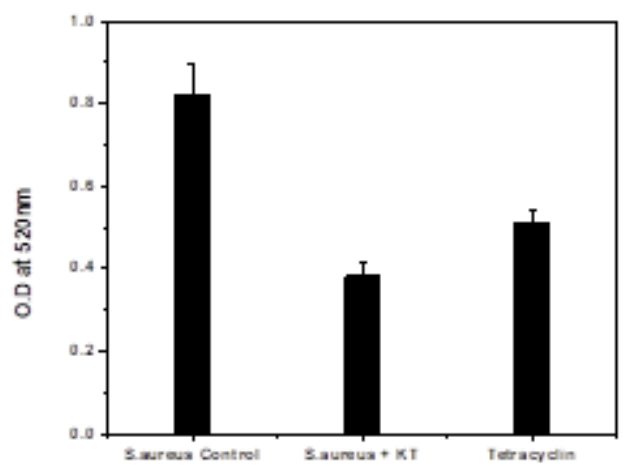

Figure 2

Reduction in quorum sensing mechanism of S. aureus biofilm as measured spectroscopically at O.D $520 \mathrm{~nm}$ on treatment with ethanolic extract of KT and standard antibiotic tetracycline. The data were of sample size $5(n=5)$ and were found to be statistically significant $(p<0.05)$.
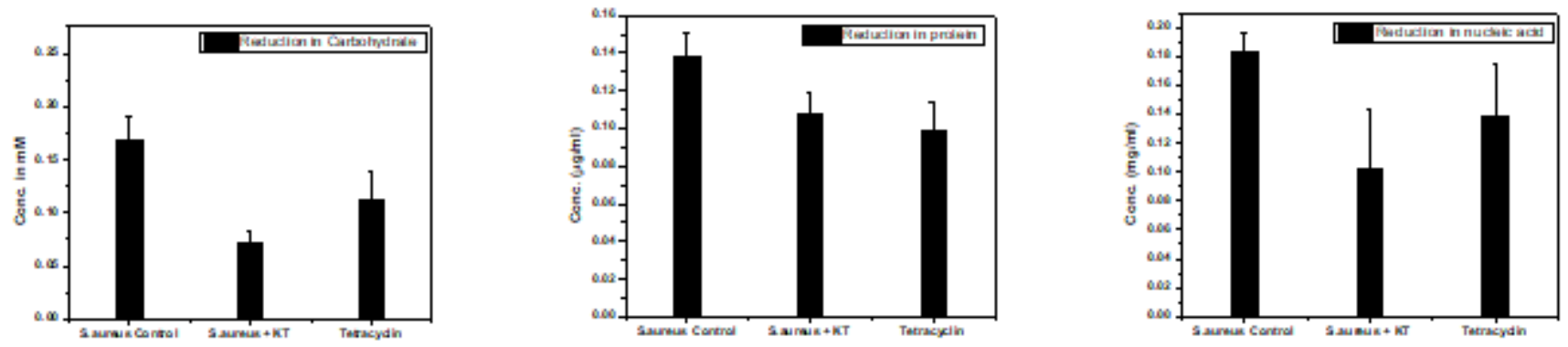

Figure 3 
Reduction in a) Carbohydrate b) protein c) nucleic acid content being present within the EPS of S. aureus upon 2 hours challenge with the ethanolic extract of KT. Results were statistically significant $(p<0.05)$.

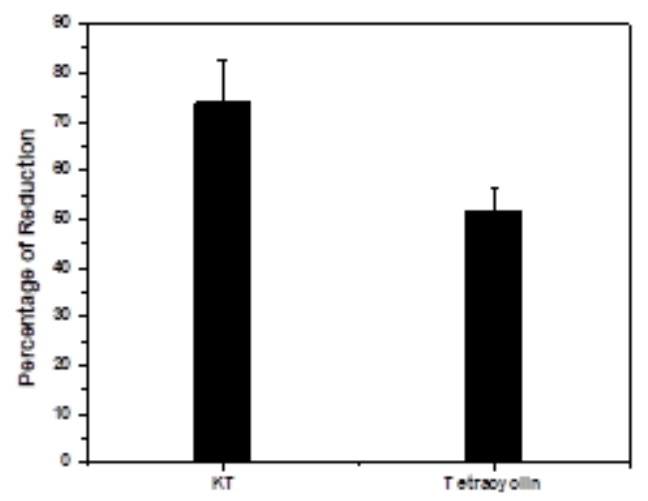

Figure 4

Eradication of biofilm formed by S.aureus in the presence of Ocimum tenuiflorum (KT)
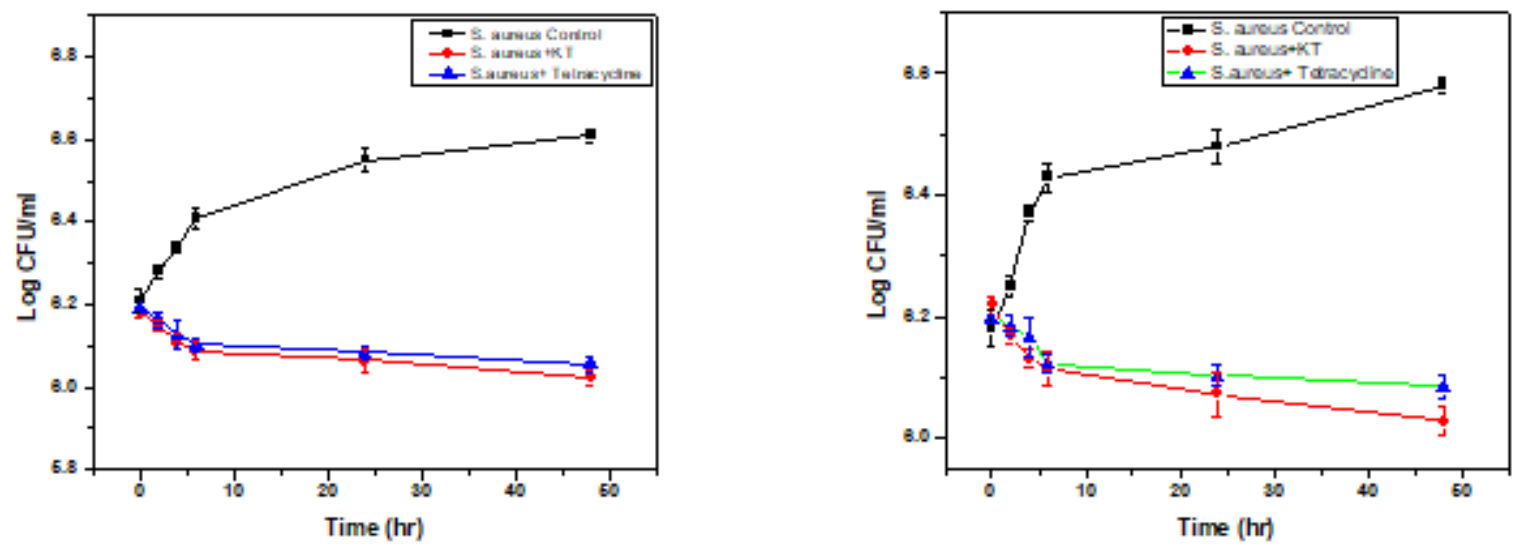

Figure 5

a) Viability count of ethanolic extract of Ocimum tenuiflorum (KT) on the biofilm forming cells of S.aureus. b) Revival of the sessile group of cells of S.aureus in the absence of challenge. The values were Mean $\pm S E$ and statistical significant with respect to control $(p<0.05)$.
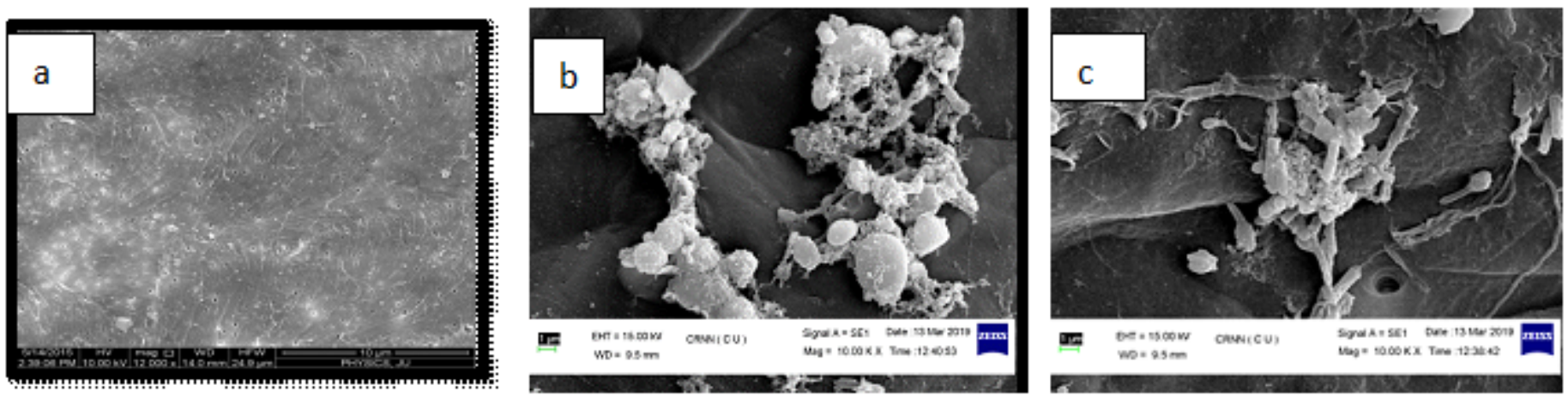
Figure 6

SEM images of biofilms developed by $\mathrm{S}$. aureus in presence of Ocimum tenuiflorum extract $(1 \mathrm{mg} / \mathrm{ml})$. a) Normal chitin flakes without any biofilm formation. b) Biofilm established on chitin flakes by S. aureus (control). c) S. aureus due to the addition of KT extract.

\section{Supplementary Files}

This is a list of supplementary files associated with this preprint. Click to download.

- Table1.docx 